\title{
Unsteady MHD Slip Boundary Layer Flow of a Nanofluid Along a Stretching Sheet
}

\author{
Falana. A. \& Temitayo L.N. \\ Department of Mechanical Engineering \\ University of Ibadan \\ Ibadan, Nigeria. \\ E-mail: falanaayode@gmail.com \\ Phone +2348037437273
}

\begin{abstract}
In this paper, we have examined the boundary layer flow of a nanofluid containing gyrostatic micro-organisms along a linearly stretching sheet in the presence of a time dependent magnetic field. The governing partial differential equations for continuity, momentum, energy, concentration of nanoparticles, and motile microorganisms density are converted into a system of the ordinary differential equations via a set of similarity transformations. These equations are numerically solved using the fourth order ordinary differential equation solver known as bvp4c in MATLAB software. The values of the shear stress at the surface which is represented by the Skin friction are compared with numerical and analytical values reported by other researchers are presented in a table and are in good agreement.
\end{abstract}

Keywords: Slip; MHD; Nanofluid; Nusselt Number; Bioconvection.

Proceedings Reference Format

Ajayi, E.A., Idris, H, Daniel, W.D. \&4Arinze, U.C. (2020): Unsteady MHD Slip Boundary Layer Flow of a Nanofluid along a Stretching Sheet. Proceedings of the 27th iSTEAMS Multidisciplinary Innovations \& Technology Transfer (MINTT) Conference. Academic City University College, Accra, Ghana. June, 2021. Pp 41-48 www.isteams.net/ghana2021. DOI - https://doi.org/ 10.22624/AIMS/iSTEAMS-2021/N27P5

\section{INTRODUCTION}

Unsteady Magneto Hydrodynamic (MHD) boundary layer flow of an electrically conducting incompressible fluid with a convective surface boundary condition is frequently encountered in many industrial and technological applications such as extrusion of plastics, the manufacture of Rayon and Nylon, the cooling of reactors, purification of crude oil, textile industry, polymer technology, and metallurgy. As a result, the simultaneous occurrence of buoyancy and magnetic field forces on fluid flow has been investigated by many researchers. MHD (Magneto-Hydrodynamics) is the science of the motion of electrically conducting fluids (Nanofluids) under the influence of applied magnetic forces (Bondareva et al., 2015).

Bio convection refers to a macroscopic convection motion of Nano fluid caused by the density gradient induced by collective swimming of motile microorganisms (Kuznetsov, 2011). 
These self-propelled motile microorganisms enhance the density of the base fluid by swimming in a particular direction, thus causing bio convection. Kuznetsov (2011) analyzed on Nano fluids containing gyrotactic microorganisms and reaffirm that the resultant large-scale motion of fluid caused by self-propelled motile microorganisms increases mixing and prevent nanoparticle agglomeration in fluids.

Heat and mass transfer in the boundary-layer flow of unsteady viscous Nano fluid along a vertical stretching sheet in the presence of magnetic field, thermal radiation, heat generation, and chemical reaction are presented by Eshetu and Shankar (2014). The authors found out that the velocity, temperature, and concentration profiles of the unsteady flow are less. than the corresponding parts of the steady state flow scenario. Kuznetsov and Nield (2010) studied analytically the free convective boundary-layer flow of a nanofluid past a vertical plate. Their results showed that the reduced Nusselt number is a decreasing function of each of buoyancy force parameter, Brownian motion parameter and thermophoresis parameter.

According to Aziz (2010), the concept of no-slip condition at the boundary layer is no longer valid for fluid flows in micro electromechanical systems and must be replaced by slip condition. Many researchers studied the effect of linear momentum and nonlinear slip on the MHD boundary layer flow with heat/mass transfer of free/forced/combined convection past different geometries. In spite of the importance of MHD related studies on boundary layer flow problems, the possibility of fluid exhibiting apparent slip phenomenon on the solid surface has received little attention.

This reveals that more work still needs to be done on unsteady boundary layer flow of Nano fluid under slip condition. Thus, the present study investigates the combined effects of velocity slip, temperature slip, bio convection Lewis number, and magnetic field and unsteadiness parameters on boundary layer flow with heat and mass transfer characteristics of a nanofluid containing oxystatic microorganisms over a stretching sheet. 


\section{MATHEMATICAL FORMULATION}

The flow is governed by the continuity equation, the momentum equation, energy equation, concentration and the conservation equations which are listed respectively below.

$$
\begin{aligned}
& \frac{\partial u}{\partial x}+\frac{\partial v}{\partial y}=0 \\
& \frac{\partial u}{\partial t}+u \frac{\partial u}{\partial x}+v \frac{\partial}{\partial} \frac{u}{y}=v \frac{\partial^{2} u}{\partial y^{2}}-\frac{O B B_{0}^{2} u}{\rho}
\end{aligned}
$$

$\underline{\partial T} \quad \underline{\partial T} \quad \underline{\partial T} \quad k \quad \partial^{2} T \quad \underline{v}^{\partial u}{ }^{2} \quad \underline{\partial C \partial T} \quad D_{t} \underline{\partial T}^{2}$

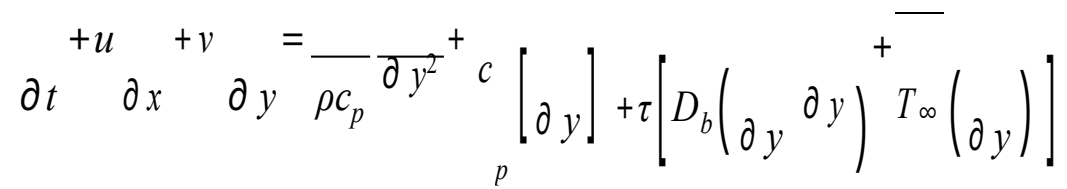

$\underline{\partial C} \quad \underline{\partial C} \quad \underline{\partial C} \quad \partial^{2} C \quad D_{T} \quad \partial^{2} T$

$$
\partial t^{+u} \partial x+{ }^{+v} \partial y=D_{B} \partial y^{2} T_{\infty}\left[\begin{array}{l}
\partial y^{2}
\end{array}\right]
$$

$\underline{\partial n} \quad \underline{\partial n} \quad \underline{\partial n} \quad b W_{c} \quad \underline{\partial} \quad \underline{\partial C} \quad \partial^{2} n$

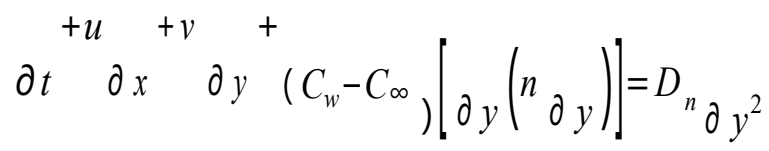

Where

$\mathrm{t}$ is time, $\mathrm{u}$ and $\mathrm{v}$ are the velocity components, $\mathrm{x}$ and $\mathrm{y}$ are the Cartesian coordinates

$T$ is the temperature, DT is the thermophoresis diffusion coefficient

$\mathrm{BD}$ is the Brownian diffusion coefficient, $\emptyset$ is the nanoparticle volume fraction $\mathrm{n}$ is the density of motile microorganisms, $\mathrm{u}$ is the kinematic viscosity $\rho$ is the density of the fluid $\sigma B$ is the fluid electrical conductivity, $B 0$ is the strength of magnetic field, $k$ is the thermal conductivity, $c p$ is the specific heat at constant pressure, $\mathrm{Dn}$ is the diffusivity of microorganisms, $b$ is the chemo taxis constant, and WC is maximum cell swimming speed. 
The initial and boundary conditions are taking as:

$$
\begin{aligned}
& t=0, u=u_{w}(x, t), T=T_{\infty}, v=0, C=C_{\infty}, n=n_{\infty} \text { everywhere } \\
& t \geq 0, u=0, v=0, T=T_{\infty}, C=C_{\infty}, n=n_{\infty} \text { all at } x=0 \\
& y=0, u=u_{w}+N \frac{\partial u}{\partial y}, T=T_{w}(x, t)+D \frac{\partial u}{}, C=C_{w}, n=n_{w} \\
& u=0, v=0, T \rightarrow T_{\infty}, C \rightarrow C_{\infty}, n \rightarrow n_{\infty}, y \rightarrow \infty
\end{aligned}
$$

Where

$$
u_{w}(x, t)=\frac{a x}{(1-\lambda t t)}, T w_{w}(x, t)=T_{\infty}+\frac{b x}{(1-\lambda t t)}, B(t)=\frac{B_{0}}{(1-\lambda t t)^{2}}
$$

We introduce the stream function $\psi x$, it) such that:

$$
u=\frac{\partial \psi}{\partial y}, v=\frac{\partial \psi}{\partial x}
$$

The following similarity) such that transformations are used in equations (1) - (5) and the boundary conditions

$$
\eta=\sqrt{\frac{a}{v(1-\lambda t t)}} y, \psi=\sqrt{\frac{a v}{1-\lambda t t}} x f(\eta), \theta(\eta)=\frac{T-T_{\infty}}{T_{w}-T_{\infty}}, \varphi(\eta)=\frac{C-C_{\infty}}{C_{w}-C_{\infty}}, \chi(\eta)=\frac{n-n_{\infty}}{n_{w}-n_{\infty}}
$$

The above equations are partial differential equations and cannot be solved numerically just like that, it has to be transformed into ordinary differential equations by similarity transformation. Thus, similarity transformations are the transformations by which an $n$-independent variable partial differential system can be converted to a system with $n-1$ independent variables. After successful transformations of all the equations along with the boundary conditions, the following equations are obtained.

Momentum $f^{\prime \prime \prime}+f f^{\prime \prime}-f^{\prime 2}-M f^{\prime}-A\left(f^{\prime}-\frac{\eta}{2} f^{\prime \prime}\right)=0$ 
$\operatorname{Energy} \theta^{\prime \prime}+i\left(f^{\prime \prime 2}\right)+\operatorname{Pr} N b \square^{\prime} \theta^{\prime}+\operatorname{Pr} N t \theta^{\prime 2}+\operatorname{Pr} \theta^{\prime}\left(f-A \frac{\eta}{2}\right)=0$

Nanoparticle concentration

$\square^{\prime \prime}+\frac{N t}{N b} \theta^{\prime \prime}+\square^{\prime} \leq\left(f-A \frac{\eta}{2}\right)=0$

Conservation for microorganism

$\chi^{\prime \prime}+\chi^{\prime} L b\left(f-\frac{\eta}{2}\right)-P e\left[\chi^{\prime} \square^{\prime}+(\chi+\Omega) \square^{\prime \prime}\right]=0$

Boundary conditions

$$
\begin{aligned}
& f(0)=0, f^{\prime}(0)=1+\alpha f^{\prime \prime}, \theta(0)=1+\gamma \theta^{\prime},(0)=1, \chi(0)=1 \\
& \text { all at } \eta=0 .
\end{aligned}
$$$$
f(\infty)=f^{\prime}(\infty)=0, \theta(\infty)=0,(\infty)=0, \chi(\infty)=0 \text { as } \eta \rightarrow \infty
$$

\section{NUMERICAL SIMULATIONS}

After successful transformations, the set of resulting nonlinear differential equations is solved using Runge -Kutta fourth order algorithm along with shooting technique. A Matlab special in built function for solving first order boundary value problems using fourth order method called Bvp4c has been employed for the purpose of simulation in this work. All the equations were firstly converted into a set of first order differential equations and were then carefully coded into matlab in the differential equation function. The boundary conditions were also coded into boundary value function. Then, about nine initial guesses were made and the solutions were obtained across a mesh of ten equally spaced points with a tolerance of 10-7 and a time step of approximately 0.3 as the case maybe. The results were then validated by comparing them with published literature values.

\section{RESULTS AND DISCUSSION}

Having numerically solved the set of nonlinear ordinary differential equations resulting from the similarity transformations the different pertinent parameters of interest such as velocity and temperature slip, magnetic, bio convection Lewis number and unsteadiness parameter on the heat and mass transfer properties of the flow are fully explored. Also, parameters of interests such as Skin friction, Nusselt number, etc were obtained and presented in tables.

In order to test for the validity and accuracy of the solution, the values of the shear stress at the surface which is represented by the Skin friction are compared with numerical and analytical values reported by Aminreza et al (2012) and Sahoo and Do (2010) in Table 4.1 This table shows the numerical solution obtained by the present algorithm and the exact solution reported by Aminreza et al (2012) and Sahoo and Do (2010) are in good agreement.

Similarly, Table 4. 2 compare results for the reduced Nusselt number $\left(-\theta^{\prime}(0) i\right.$. 
Table 4.1: Comparison of results for the skin friction coefficient when $A=N t=N b=0.1, E c=P e=M$ $=\mathrm{Lb}=\mathbf{0}$, $\mathrm{Le}=\operatorname{Pr}=10$.

\begin{tabular}{|ll|l|l|c|}
\hline $1.0 \beta$ & & Aminreza et al (2012) & Sahoo and Do (2010) & Present result \\
\hline 2.0 & 0 & 1.000000 & 1.001154 & 1.001396 \\
& 0.1 & 0.872082 & 0.871447 & 0.873440 \\
& 0.2 & 0.776377 & 0.774933 & 0.777700 \\
& 0.3 & 0.701548 & 0.699738 & 0.702840 \\
& 0.5 & 0.591195 & 0.589195 & 0.592430 \\
& 1.0 & 0.430160 & 0.428450 & 0.431279 \\
& 2.0 & 0.283980 & 0.282893 & 0.284937 \\
\hline
\end{tabular}

Table 4.2: Comparison of results for the reduced Nusselt number when $L e=P r=10$, no slip condition with varying $\mathrm{Nt}$ and $\mathrm{Nb}$.

\begin{tabular}{|l|l|l|l|l|}
\hline $\mathrm{Nb}$ & $\mathrm{Nt}$ & Aminreza et al (2012) & Khan and Pop (2010) & Present result \\
\hline 0.1 & 0.1 & 0.9523768 & 0.9524 & 0.9522609 \\
0.1 & 0.2 & 0.6931743 & 0.6932 & 0.6931502 \\
0.1 & 0.3 & 0.5200790 & 0.5201 & 0.5201161 \\
0.1 & 0.4 & 0.4025808 & 0.4026 & 0.4026594 \\
0.1 & 0.5 & 0.3210543 & 0.3211 & 0.3211623 \\
0.2 & 0.1 & 0.5055814 & 0.5056 & 0.5055271 \\
0.3 & 0.1 & 0.2521560 & 0.2522 & 0.2521323 \\
0.4 & 0.1 & 0.1194059 & 0.1194 & 0.1193960 \\
0.5 & 0.1 & 0.0542534 & 0.0543 & 0.0542494 \\
\hline
\end{tabular}




\section{CONCLUSIONS}

In this paper, we have examined the boundary layer flow of a nanofluid containing gyrotactic micro-organisms along a linearly stretching sheet in the presence of a time dependent magnetic field. These equations are numerically solved using the fourth order ordinary differential equation solver known as bvp4c in MATLAB software. The results show the following conclusions: The local skin friction coefficient increases with the simultaneous increase of the magnetic and unsteadiness parameter. The Local Nusselt number is decreasing with a simultaneous increase of magnetic and unsteadiness parameter.

\section{REFERENCES}

1. Aminreza, N., Rashid P, and Mohammad G. (2012). Effect of partial slip boundary condition on the flow and heat transfer of nanofluids past stretching sheet prescribed constant wall temperature. International Journal of Thermal Sciences.54:253-261.

2. Aziz, A. (2010). Hydrodynamic and thermal slip flow boundary layers over a flat plate with constant heat flux boundary condition. Communications in nonlinear Science and Numerical simulation.15 (3):573-580.

3. Bachok, N., Ishak, A. and Pop, I. (2010). Boundary Layer Flow of Nanofluids over a Moving Surface in a Flowing Fluid. International Journal of Thermal Sciences.49:1663-1668.

4. Bondareva, N.S., Sheremet, M.A. and Pop, I. (2015). Magnetic Field Effect on the Unsteady Natural Convection in a Right-Angle Trapezoidal Cavity Filled with a Nanofluid. InternationalJournal of Numerical Methods for Heat \& Fluid Flow.25:1924-1946.

5. Fekry, M. H, Mahdy, M., Ramadan, A. M., Omima, A. and Abo, Z.(2016). Effects of Viscous Dissipation on Unsteady MHD Thermo Bioconvection Boundary Layer Flow of a NanofluidContaining Gyrotactic Microorganisms along a Stretching Sheet.World Journal of Mechanics.6:505-526.

6. Ferdows, M., Khan, M.S., Alam, M.M. and Sun, S. (2012). MHD Mixed Convective Boundary Layer Flow of a Nanofluid through a Porous Medium Due to an Exponentially StretchingSheet.Mathematical Problems in Engineering, 2012. Article ID: 408528.

7. Ferdows, M., Khan, M.S., Bég, O.A., Alam, M.M. and Azad, M.A.K. (2013). Numerical Study of Transient MagnetohydrodynamicRadiative Free Convection Nanofluid Flow

8. from a Stretching Permeable Surface.Journal of Process Mechanical Engineering,1-16.

9. Gorla, R.S.R. and Hossain, A. (2013).Mixed Convective Boundary Layer Flow over a Vertical Cylinder Embedded in a Porous Medium Saturated with a Nanofluid. International Journal of Numerical Methods for Heat \& Fluid Flow. 23:1393-1405.

10. Haile, E. and Shankar, B. (2014). Heat and Mass Transfer in the Boundary Layer of Unsteady Viscous Nanofluid along a Vertical Stretching Sheet. Journal of Computational Engineering,2014. Article ID: 345153.

11. Hady, F.M., Mohamed, R.A., Mahdy, A. and Abo Zaid, O.A. (2016). Non-Darcy Natural Convection Boundary Layer Flow over a Vertical Cone in Porous Media Saturated with aNanofluid Containing Gyrotactic Microorganisms with a Convective Boundary Condition.Journal of Nanofluids.5:765-773.

12. Khan, W.A. and Pop, I. (2010).Boundary-Layer Flow of a Nanofluid past a Stretching Sheet. International Journal of Heat and Mass Transfer .53: 2477-2483. 
13. Kuznetsove, A.V. and Avramenko, A.A. (2003). Stability Analysis of Bioconvection of Gyrotactic Motile Microorganisms in a Fluid Saturated Porous Medium. Transport in Porous Media.53:95-104.

14. Kuznetsov, A.V. (2006). The Onset of Thermo-Bioconvection in a Shallow Fluid Saturated Porous Layer Heated from Below in a Suspension of Oxytactic Microorganisms. European Journal of Mechanics-B/Fluids.25: 223-233.

15. Kuznetsov, A. and Nield, D. (2010). Natural Convective Boundary-Layer Flow of a Nanofluid past a Vertical Plate. International Journal of Thermal Sciences. 49:243-247.

16. Kuznetsove, A.V. (2006). Investigation of the Onset of Bioconvection in a Suspension of Oxytactic Microorganisms Subjected to High-Frequency Vertical Vibration. Theoreticaland Computational Fluid Dynamics.20: 73-87.

17. Mahdy, A. (2016). Natural Convection Boundary Layer Flow Due to Gyrotactic Microorganisms about Vertical Cone in Porous Media Saturated by a nanofluid. Journal of the Brazilian Societyof Mechanical Sciences and Engineering.38: 67-76.

18. Makinde, O.D. (2012). Effects of Viscous Dissipation and Newtonian Heating on Boundary- Layer Flow of Nanofluids over a Flat Plate. International Journal of Numerical Methods for Heat and Fluid Flow.23:1291-1303.

19. Mustafa, M., Hayat, T. and Obaidat, S. (2013). Boundary Layer Flow of a Nanofluid over an Exponentially Stretching Sheet with Convective Boundary Conditions. International Journal ofNumerical Methods for Heat \& Fluid Flow. 23:945-959.

20. Nadeem, S. and Lee, C. (2012). Boundary Layer Flow of Nanofluid over an Exponentially Stretching Surface. Nanoscale Research Letters.7: 94-99.

21. Na, T.Y. and Pop, I. (1996). Unsteady Flow past a Stretching Sheet. Mechanics Research Communications.23:413-422.

22. Sahoo, B. and Do, Y. (2010). Effects of slip on sheet-driven flow and heat transfer of a third grade fluid past a stretching sheet, Int. Commun. Heat Mass Transfer 37: 1064e1071.

23. Sun, Q. and Pop, I. (2011). Free Convection in a Triangle Cavity Filled with a Porous Medium Saturated with Nanofluids with Flush Mounted Heater on the Wall. International Journal of Thermal Sciences.50: 2141-2153.

24. Shehzad, S.A., Abdullah, Z., Abbasi, F.M., Hayat, T. and Alsaedi, A. (2016). Field Effect in ThreeDimensional Flow of an Oldroyd-B Nanofluid over a Radiative Surface. Journal Of Magnetism and Magnetic Materials.39: 97-108.

25. Uddi, M.J., Khan, W.A. and Ismail, A.I. (2012). Effect of Dissipation on Free Convective Flow of a Non-Newtonian Nanofluid in a Porous Medium with Gyrotactic Microorganisms. Journal of Nano engineering and Nanosystems.227:11-18 